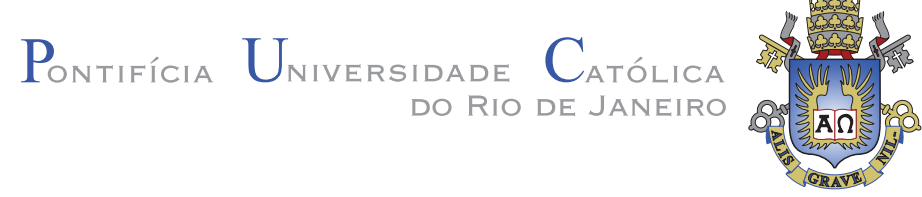

Miriam Cardoso Costa

\title{
Análise da Eficiência de Operadores de Logística no Mercado Brasileiro
}

Dissertação apresentada como requisito parcial para obtenção do grau de Mestre pelo Programa de Pós-Graduação em Engenharia de Produção do Departamento de Engenharia Industrial da PUC-Rio

Orientador Prof. Madiagne Diallo

Co-Orientador: Sr. Luis Eduardo Madeiro Guedes 


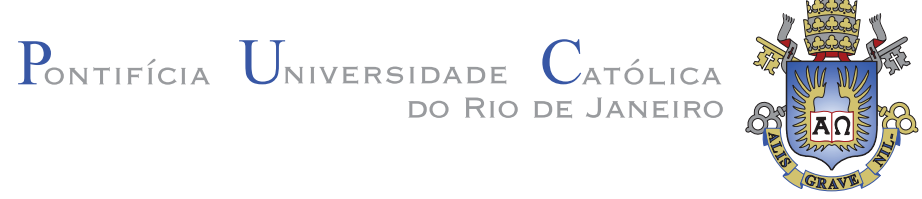

Miriam Cardoso Costa

\section{Análise da Eficiência de Operadores de Logística no Mercado Brasileiro}

Dissertação apresentada como requisito parcial para obtenção do grau de Mestre pelo Programa de Pós-Graduação em Engenharia de Produção do Departamento de Engenharia Industrial do Centro Técnico Científico da PUC-Rio. Aprovada pela Comissão Examinadora abaixo assinada.

Sr. Luis Eduardo Madeiro Guedes

Co-Orientador

Overview Pesquisa

Prof. Fernanda Maria Pereira Raupp

Departamento de Engenharia Industrial - PUC-Rio

Prof. Nélio Domingues Pizzolato

Departamento de Engenharia Industrial - PUC-Rio

Prof. José Eugenio Leal

Coordenador Setorial do Centro Técnico Científico - PUC-Rio

Rio de Janeiro, 25 de Agosto de 2010 
Todos os direitos reservados. É proibida a reprodução total ou parcial do trabalho sem autorização da universidade, do autor e do orientador.

\section{Miriam Cardoso Costa}

Bacharel em Ciências Estatísticas na Escola Nacional de Ciências Estatísticas (ENCE)

Ficha Catalográfica

Costa, Miriam Cardoso

Análise da Eficiência de Operadores de Logística no Mercado Brasileiro / Miriam Cardoso Costa; orientador: Madiagne Diallo; co-orientador: Luis Eduardo Madeiro Guedes. - 2010.

v., 76 f: il. ; $30 \mathrm{~cm}$

1. Dissertação (Mestrado) - Pontifícia Universidade Católica do Rio de Janeiro, Departamento de Engenharia Industrial.

Inclui referências bibliográficas.

1. Engenharia Industrial - Teses. 2. Operadores de logística. 3. DEA. 4. Tobit. I. Diallo, Madiagne. II. Guedes, Luis Eduardo Madeiro. III. Pontifíca Universidade Católica do Rio de Janeiro. Departamento de Engenharia Industrial. IV. Título. 


\section{Agradecimentos}

\section{A Deus.}

À minha mãe, pela minha educação e por estimular os meus estudos.

À minha irmã, meu Bichinho, que colaborou digitando a base de dados.

Ao Leonardo, pelo carinho, pela compreensão e por sempre estar ao meu lado.

Ao professor Madiagne, pela orientação.

Ao Luis Eduardo, pela coorientação.

Ao professor Luis Felipe, pela colaboração.

Aos colegas do curso e funcionários da secretaria do departamento, em especial à Claudia.

Por fim, ao CNPq, pela bolsa. 


\section{Resumo}

Costa, Miriam Cardoso; Diallo, Madiagne; Guedes, Luis Eduardo Madeiro. Análise da Eficiência de Operadores de Logística no Mercado Brasileiro. Rio de Janeiro, 2010. 76p. Dissertação de Mestrado - Departamento de Engenharia Industrial, Pontifícia Universidade Católica do Rio de Janeiro.

O trabalho tem como principal objetivo avaliar a eficiência de operadores de logística que atuam no mercado brasileiro. Dentro desse contexto, serão avaliadas as empresas listadas na Revista Tecnologística de junho de 2008. Serão sugeridas soluções para as questões ligadas ao foco das empresas e as tendências do mercado de operações de logística. O estudo deverá destacar as variáveis que têm impacto positivo e negativo na eficiência dos operadores, e propor soluções para os problemas de ineficiência. A metodologia de Análise Envoltória de Dados será utilizada para eliminar o efeito escala, enquanto o modelo tobit balizará a avaliação do impacto das variáveis selecionadas no mercado.

\section{Palavras-chave}

Operadores de logística; DEA; Tobit. 


\section{Abstract}

Costa, Miriam Cardoso; Diallo, Madiagne; Guedes, Luis Eduardo Madeiro. Analysis of the efficiency of logistics operators in brazilian market. Rio de Janeiro, 2010. 76p. MSc Dissertation - Departamento de Engenharia Industrial, Pontifícia Universidade Católica do Rio de Janeiro.

The study has as its main objective to evaluate the efficiency of logistics operators in the Brazilian market. Within this context, we will evaluate the companies listed in the June 2008 edition of the Tenologística Magazine. Solutions regarding the focus of business and market trends of logistics operations will be suggested. The study will highlight the variables that have positive and negative impact on the efficiency of operators and propose solutions to inefficiency problems. Data Envelopment Analysis will be used to eliminate scale effect, and the tobit model to assess the impact of selected variables on the market.

\section{Keywords}

Logistics operators; DEA; tobit. 


\section{Sumário}

$\begin{array}{lll}1 & \text { Introdução } & 10\end{array}$

2 Operadores de Logística $\quad 13$

2.1 Histórico 13

2.2 Conceito e segmentação 14

2.3 Caracterização dos serviços logísticos 17

$\begin{array}{ll}2.4 & \text { Medida do desempenho logístico } \\ & 18\end{array}$

3 Descrição da Metodologia $\quad 20$

3.1 Estratégia de Análise 20

3.2 Análise Envoltória de Dados (DEA) 20

3.3 Modelo tobit 33

3.4 Aplicação de DEA e tobit 40

4 Aspectos Iniciais da Ánalise $\quad 42$

4.1 Descrição da base 42

4.2 Variáveis selecionadas 43

4.3 Tratamento dos dados 44

5 Análise dos Resultados $\quad 46$

5.1 Os operadores de logísticas em estudo 46

5.2 Primeiro Estágio $\quad 52$

5.3 Segundo Estágio $\quad 55$

6 Conclusões e Trabalhos Futuros $\quad 60$

$\begin{array}{ll}\text { Referências Bibliográficas } & 63\end{array}$

$\begin{array}{lll}\text { A Tratamento dos dados } & 67\end{array}$

B Análise dos Dados para Aplicação do Modelo DEA 68

$\begin{array}{ll}\text { C Iteração do Modelo BCC-I } & 70\end{array}$

D $\quad$ Modelos de regressão tobit ajustados $\quad 72$ 


\section{Lista de figuras}

3.1 Regressão linear 23

3.2 Conjuto de dados 24

3.3 Fronteira de Eficiência 25

3.4 Fronteira de Eficiência 25

3.5 Representação Gráfica CCR-O 28

3.6 Representação Gráfica CCR-I 29

3.7 Representação Gráfica BCC-O 31

3.8 Representação Gráfica BCC-I 32

3.9 Resumo dos estudos com DEA e regressão tobit até 2004

5.1 Tempo de mercado (anos) 47

5.2 Certificações 48

5.3 Serviços Oferecidos $\quad 49$

5.4 Transporte Oferecido 49

5.5 Tecnologia Empregada 50

5.6 Porcentagem das empresas que possuem frota própria de transporte 51

5.7 Distribuição da tecnologia de rastreamento por tipo de frota 51

5.8 Distribuição do tipo de tecnologia de rastreamento 52

5.9 Boxplot das eficiências obtidas através do modelo BCC-I 54

5.10 Ordenação dos score de eficiência obtidos pelo modelo BCC-I 55 


\section{Lista de tabelas}

2.1 Evolução da logística 13

3.1 Exemplo de saída do modelo tobit 38

4.1 Variáveis disponível na Revista Tecnologística 43

4.2 Análise dos dados 44

4.3 Análise dos dados tratados $\quad 45$

5.1 Origem dos operadores de logística 47

5.2 Score das eficiências do modelo BCC-I 53

5.3 Participação Virtual das eficiências dos operadores de logística para o modelo BCC-I 54

5.4 Ajuste do modelo de regressão tobit selecionado 56

B.1 Dados utilizados na aplicação da metodolgia DEA 69

C.1 Participação virtual do modelo BCC-I 71

D.1 Tentativa de ajuste do modelo de regressão tobit 72

D.2 Tentativa de ajuste do modelo de regressão tobit 73

D.3 Tentativa de ajuste do modelo de regressão tobit 73

D.4 Tentativa de ajuste do modelo de regressão tobit 74

D.5 Tentativa de ajuste do modelo de regressão tobit 74

D.6 Tentativa de ajuste do modelo de regressão tobit 75

D.7 Tentativa de ajuste do modelo de regressão tobit $\quad 75$

D.8 Tentativa de ajuste do modelo de regressão tobit 76 